\title{
Note on Statistics and Transliteration
}

This book was written while I was on leave from teaching in 2009-2010. The statistics, therefore, reflect updated statistics at that time. Epidemiological statistics and statistics on public health programs are constantly changing, but I believe that the statistics included in this book from 2009-2010 accurately depict the current trends as of 2012, when the book manuscript went in to press.

I have chosen not to use diacritical marks for transliteration of non-English words to facilitate reading. 
